\title{
Machine Learning-Based Optoacoustic Tissue Classification Method for Laser Osteotomes Using an Air-Coupled Transducer
}

\author{
Hervé Nguendon Kenhagho, (1) ${ }^{1 *}$ Ferda Canbaz, (1) ${ }^{1}$ Tomas E. Gomez Alvarez-Arenas, (i) ${ }^{2}$ \\ Raphael Guzman, ${ }^{3,4}$ Philippe Cattin, $\mathbb{1}^{5}$ and Azhar Zam (iD ${ }^{1}$ \\ ${ }^{1}$ Biomedical Laser and Optics Group, Department of Biomedical Engineering, University of Basel, Gewerbestrasse 14, \\ Allschwil, 4123, Switzerland \\ ${ }^{2}$ Department of Ultrasonic and Sensors Technologies, Information and Physical Technologies Institute ITEFI, Spanish \\ National Research Council (CSIC), Serrano 144, Madrid, 28006, Spain \\ ${ }^{3}$ Brain Ischemia and Regeneration, Department of Biomedicine, University of Basel, University Hospital of Basel, Basel, \\ 4031, Switzerland \\ ${ }^{4}$ Neurosurgery Group, Department of Biomedical Engineering, University of Basel, Allschwil, 4123, Switzerland \\ ${ }^{5}$ Department of Biomedical Engineering, Center for Medical Image Analysis and Navigation, University of Basel, \\ Gewerbestrasse 14, Allschwil, 4123, Switzerland
}

Background and Objectives: Using lasers instead of mechanical tools for bone cutting holds many advantages, including functional cuts, contactless interaction, and faster wound healing. To fully exploit the benefits of lasers over conventional mechanical tools, a real-time feedback to classify tissue is proposed.

Study Design/Materials and Methods: In this paper, we simultaneously classified five tissue types-hard and soft bone, muscle, fat, and skin from five proximal and distal fresh porcine femurs-based on the laser-induced acoustic shock waves (ASWs) generated. For laser ablation, a nanosecond frequency-doubled Nd:YAG laser source at $532 \mathrm{~nm}$ and a microsecond Er:YAG laser source at $2940 \mathrm{~nm}$ were used to create 10 craters on the surface of each proximal and distal femur. Depending on the application, the Nd:YAG or Er:YAG can be used for bone cutting. For ASW recording, an air-coupled transducer was placed $5 \mathrm{~cm}$ away from the ablated spot. For tissue classification, we analyzed the measured acoustics by looking at the amplitude-frequency band of 0.11-0.27 and $0.27-0.53 \mathrm{MHz}$, which provided the least average classification error for Er:YAG and Nd:YAG, respectively. For data reduction, we used the amplitude-frequency band as an input of the principal component analysis (PCA). On the basis of PCA scores, we compared the performance of the artificial neural network (ANN), the quadratic- and Gaussian-support vector machine (SVM) to classify tissue types. A set of 14,400 data points, measured from 10 craters in four proximal and distal femurs, was used as training data, while a set of 3,600 data points from 10 craters in the remaining proximal and distal femur was considered as testing data, for each laser.

Results: The ANN performed best for both lasers, with an average classification error for all tissues of $5.01 \pm 5.06 \%$ and $9.12 \pm 3.39 \%$, using the Nd:YAG and Er:YAG lasers, respectively. Then, the Gaussian-SVM performed better than the quadratic SVM during the cutting with both lasers. The Gaussian-SVM yielded average classification errors of $15.17 \pm 13.12 \%$ and $16.85 \pm 7.59 \%$, using the Nd:YAG and Er:YAG lasers, respectively. The worst performance was achieved with the quadratic-SVM with a classification error of $50.34 \pm 35.04 \%$ and $69.96 \pm 25.49 \%$, using the Nd:YAG and Er:YAG lasers.

Conclusion: We foresee using the ANN to differentiate tissues in real-time during laser osteotomy. Lasers Surg. Med. (C) 2020 Wiley Periodicals LLC

Key words: laser ablation; tissue classification; acoustic shock signal; principal component analysis; support vector machine; artificial network machine

\section{INTRODUCTION}

Conventional osteotomy relies on mechanical tools, such as scalpels, saws, and burrs [1,2], which often result in mechanical trauma, metal debris, bacterial contamination, and collateral damage to soft tissues [1]. Major drawbacks of mechanical tools are excessive force, fractures, vibrations, and heat that can damage the surrounding tissue [3]. These side effects lead to prolonged healing periods. In contrast to conventional osteotomy, laser osteotomy (where a laser is

Conflict of Interest Disclosures: All authors have completed and submitted the ICMJE Form for Disclosure of Potential Conflicts of Interest and none were reported.

${ }^{*}$ Correspondence to: Hervé Nguendon Kenhagho, MSc or Dr. Azhar Zam, Biomedical Laser and Optics Group, Department of Biomedical Engineering, University of Basel, Gewerbestrasse 14, Allschwil, Switzerland.

E-mail: herve.nguendon@unibas.ch (H.N.K.); E-mail: azhar.zam@unibas.ch (A.Z.)

Accepted 14 June 2020

Published online in Wiley Online Library

(wileyonlinelibrary.com).

DOI $10.1002 / \mathrm{sm} .23290$ 
used to cut the bone) has emerged and evolved in recent years to achieve precision cutting, sterility, and reduced trauma during surgery, followed by fast healing times [4]. Therefore, laser technologies appear to offer a sophisticated solution to overcome the disadvantages of mechanical tools $[5,6]$. The laser surgery process, which has been considered most effective for bone tissue uses an erbium-doped yttrium aluminum garnet (Er:YAG) laser source operating at $2940 \mathrm{~nm}[7,8]$. The reason being that the operation wavelength of the Er:YAG laser corresponds to one of the highest absorption peak of water and hydroxyapatite, the main component of bone [9]. However, this operation wavelength with microsecond pulse duration leads to ablation by photothermal vaporization $[10,11]$. To decrease the effects of photo-thermal vaporization, which may result in carbonization and surface roughness during laser cutting, water cooling, or spraying systems (wet environment) are widely used $[9,10]$. With an appropriate water-cooling system, the Er:YAG laser can be used to achieve greater ablation depth and better surface morphology $[10,12,13]$. This is because water prevents pulpal heating and dehydration, which are the primary causes of thermal damage and reduced tissue ablation [10,14,15]. Such laser assisted and water-cooled systems showing efficient ablation rates while producing early carbonization have been presented in the literature $[7,16,17]$.

In contrast to the microsecond Er:YAG laser, the nanosecond neodimium-doped yttrium aluminum garnet (Nd:YAG) laser source results in a plasma-based ablation $[5,18,19]$. Ablation with a nanosecond pulse duration is characterized by a combination of nonlinear absorption and Coulomb explosion without any significant temperature increase to the surroundings in wet environment [19-21]. This is because a very high rate of pulse energy is transformed into heat in the liquid-containing tissue [22]. Therefore, the thermal confinement condition is fulfilled. In other words, in a wet environment, a nanosecond pulse heats tissue more rapidly than the time it requires for the thermoelastic expansion of heated volume to occur [23]. These radiation conditions are known as confined-thermal conditions in which thermal heat does not spread out of the irradiation volume during the time of heat production by the laser pulse [24]. Furthermore, in contrast to the $2940 \mathrm{~nm}$, the wavelength of $532 \mathrm{~nm}$ is transparent in water and seems to be well suited for tissue ablation with a substantial water layer such as in knee arthroscopy $[9,18]$. However, at a wavelength of $1064 \mathrm{~nm}$, water has a higher absorption coefficient providing less penetration depth compared with the wavelength of $532 \mathrm{~nm}$. In other words, in a wet environment, energy absorption at a wavelength of $532 \mathrm{~nm}$ is very low compared to that of $1064 \mathrm{~nm}$ or $2940 \mathrm{~nm}[9,18]$.

Acoustic shock waves (ASWs) are pressure waves produced due to the rapid release of energy when a material is exposed to mechanical or thermal influences. Interaction with laser light also produces ASWs during the ablation process $[20,25]$. The ASW propagates as a spherical wavefront, which is measured using acoustic emission sensors, such as piezoelectric transducers (PZTs) and air-coupled transducers (ACTs) (microphones), which convert the spherical wavefront into electrical signals [26-28]. PZTs combined with a matching gel or water were used in direct contact acoustic detection to avoid the impedance mismatch with air. Furthermore, the high attenuation of shock waves in the air of $1.6 \mathrm{~dB} / \mathrm{cm}$ for $1 \mathrm{MHz}$ frequency components also contributes to challenges in detecting ASW signals without direct contact [29]. To improve the mismatch of non-contact ASW detection, ACTs were used. This is because the ACTs have a fundamentally low mechanical impedance mismatch with the air inducing broader bandwidth and good signal-tonoise ratio. This better acoustic coupling abolished the need for complex matching layers, which was generally used in PZTs [30]. However, the signal-to-noise ratio that is provided by ACTs is still less than the PZTs in direct contact.

The features of the ASWs generated are governed by the laser pulse's parameters such as the laser energy and the focusing conditions. However, the ASW mainly depends on the type of material (i.e., hard tissue or soft tissue) being cut [31]. Hence, by analyzing the generated ASWs, tissue types can be classified. Previously, ASWs measured were already used for photothermal therapy such as temperature monitoring during radiofrequency ablation; and forming lesion control in real-time [32-34]. Additionally, ASWs were also investigated for incision depth controlled during laser ablation $[22,35]$. But, only knowing the temperature and the depth of the incision is not enough. We also want to know which tissue type we are cutting. The method used to classify tissue types based on the ASW emitted can be performed using intensive computational methods such as machine learning. The main reason was, machine leaning combined with acoustic emission sensors were already well established in industry and pre-clinical applications [12,22,35-38]. Other studies demonstrated that acoustic waves can be separated into multiple frequency bands for optoacoustic segmentation and visualization using a truncated k-space [39]. On the basis of this method, the efficiency of image artefacts was better than with zero-padding. We also considered the potential usage of such approaches in classification workflow. Therefore, characterization of frequency band of acoustic waves measured by ACTs were combined with machine learning methods for optoacoustic feedback in laser osteotomy.

Support vector machines (SVMs) represent a major development in pattern recognition for classification [40,41]. SVMs can find a hyperplane that divides samples into two classes with the widest margin between them. Additionally, SVMs extend this concept to a higher dimensional setting using a kernel function to illustrate a similarity measure in the experimental setup [40]. Both innovations can be formulated in a quadratic or Gaussian function framework, whose optimum solution is obtained in the computation time of a polynomial or a radial basis function kernel, respectively [42]. Therefore, SVMs are effective and practical solutions for biomedical signal recognition [40,41].

Another approach to classify tissue types is to use the artificial neural network (ANN) [43]. The ANN is a machine learning that is composed of an input layer, hidden layers that represent features, and an output layer [44]. ANN is a nonlinear model that is easy to use and 
understand compared with statistical methods. The reason being that ANN is a nonparametric model, while most of the statistical methods are parametric models that need a higher background of statistics. ANN with the back propagation learning algorithm is widely used in solving various classification and forecasting problems [45,46].

In this study, we ablated hard bone, soft bone, muscle, fat, and skin tissues, using a nanosecond Nd:YAG laser at $532 \mathrm{~nm}$ and a microsecond Er:YAG laser at $2940 \mathrm{~nm}$. We measured the emitted ASWs using a high-efficiency broad-band air-coupled piezoelectric ultrasonic transducer. To simultaneously classify tissue types, we used and compared the performances of principal component analysis (PCA) combined with either a quadratic/ Gaussian-SVM or the ANN. Here, PCA was used for data reduction to decrease the computational time during tissue classification [47]. To the best of our knowledge, our group was the first one to use these machine learning methods to investigate optoacoustic tissue classification.

\section{MATERIAL AND METHOD}

\section{Sample Preparation}

In the laser-tissue ablation experiments, we used five fresh porcine proximal and distal femurs. Each fresh porcine proximal and distal femur was purchased at a local butcher each day. With scalpels, the connective tissues (Fig. 1) were carefully separated to extract hard and soft bone, muscle, fat, and skin tissues from each proximal and distal femur. The sample was then rinsed in distilled water before the laser experiments. The dimensions of all compact bone fragments, soft bone, muscle, fat, and skin tissues were $10 \times 50 \times 5 \mathrm{~mm}^{3}$.

\section{Experimental Set-Up}

The laser ablation experiments were conducted with different samples in wet conditions. A spray of distilled water with a flow rate of $0.1 \mathrm{ml} / \mathrm{s}$ was directed to the spot of ablation, wetting the sample each time, before the laser pulse hit the tissue. Ablation was performed using a Q-switched frequency-doubled Nd:YAG laser (Q-smart 850; Quantel, Paris, France) at $532 \mathrm{~nm}$ (producing 5 nanoseconds pulses) and an Er:YAG laser (litetouch LI-FG0001A; Syneron Candela, Syneron, Israel) at $2940 \mathrm{~nm}$ (producing 400 microseconds pulses) (Fig. 2a and b). The output pulse energy of the Nd:YAG laser and the Er:YAG laser were 200 and $940 \mathrm{~mJ}$, respectively.

The triggering signal was collected directly from the $\mathrm{Nd}$ :YAG laser. In the case of Er:YAG, a $\mathrm{CaF}_{2}$ window was placed in front of the laser head to split the incident laser beam into two parts-96\% transmitted and 4\% reflected light- to allow for a triggering signal. The reflected light was collected by a fast PbSe photodiode (PbSe Fixed Gain Detector, PDA20H, 1500-4800 nm; Thorlabs, Munich, Germany). For both systems, a data recording element was embedded in a single opto-acoustic system (Fig. 2a and $b$ ). The trigger signal activates the capturing of the signal received by the transducer. In the experiments, data recording took place during a time window-also known as the data acquisition window for each acoustic wave-of 0.82 milliseconds. This time window was determined based on the measured acoustic signal where a high signal-to-noise ratio was obtained. A corner mirror placed in the beam path of the laser was used to reflect the laser pulse at a $90^{\circ}$ angle. The output beam of the laser was then focused on the surface of the target specimen by using a $30 \mathrm{~mm}$ lens in each experiment. Several craters were produced ex vivo on the specimens, using the two lasers. For both lasers, each sample was exposed to 180 laser pulses at a repetition rate of $2 \mathrm{~Hz}$ at a single location. This procedure was repeated at 10 different ablation locations, spaced $4 \mathrm{~mm}$ apart. Hence, the number of craters done in each experiment was 500 craters -20 craters at the surface of the same tissue type extracted from each proximal and distal femur.

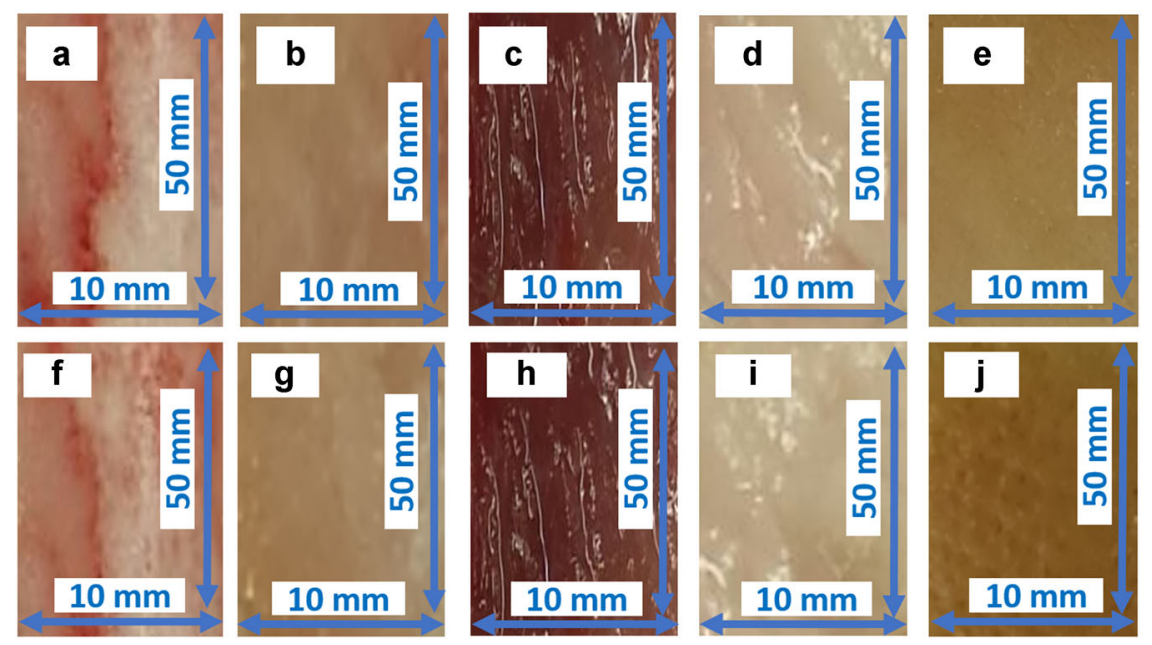

Fig. 1. Tissue samples from one fresh porcine femur. Proximal femur: hard bone (a), soft bone (b), muscle (c), fat (d), and skin (e); distal femur: hard bone (f), soft bone (g), muscle (h), fat (i), and skin $(\mathbf{j})$. 

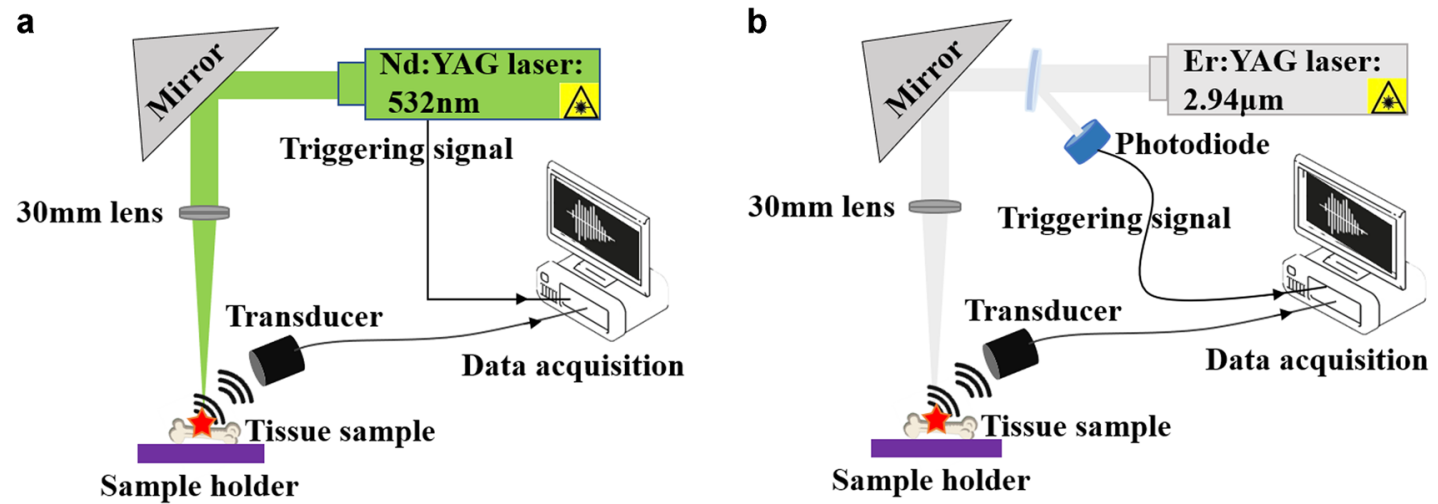

Fig. 2. Schematic of the experimental set-up for the laser-induced acoustic shock wave measurement using (a) the nanosecond Q-switched Nd:YAG laser and (b) the microsecond Er:YAG laser during tissue ablation. Er:YAG, erbium-doped yttrium aluminum garnet; Nd:YAG, neodimium-doped yttrium aluminum garnet.

The ASW is radiated from the ablation spot into the surrounding media and is picked up by the transducer, where the acoustic wave is converted into an electrical signal [20].

\section{Detection and Analysis of the ASW Signal}

This transducer was a self-developed, custom-made aircoupled PZT (manufactured in the ITEFI-Instituto de Technologias Fisicas y de la Information, CSIC, Madrid, Spain), with a resonance frequency of $0.4 \mathrm{MHz}$, an available frequency band of $0.1-0.8 \mathrm{MHz}$, and a $15 \mathrm{~mm}$ aperture (Fig. 3). The calibration of the frequency band (frequency response) of the transducer in reception mode was obtained by using a calibrated source with flat frequency response in the frequency range $0.1-1 \mathrm{MHz}$. Being in the low $\mathrm{MHz}$ range compared with conventional microphones, the design and fabrication of air-coupled PZTs is complicated due to the enormous impedance mismatch between the piezoelectric element and air. For our application, an optimized stack of detuned quarter wavelength matching layers was used to optimize both transducer bandwidth and sensitivity that are critical in this application $[48,49]$. The temporal profile of ASW signals detected by the transducer were amplified by $30 \mathrm{~dB}$ and digitized by a PCI Express x8 (16-bit resolution, four channels at $10 \mathrm{MS} /$ $\mathrm{s}$ each, M4i.44xx-x8; Spectrum Microelectronic GmbH, Grosshansdorf, Germany). The transducer was placed at a $45^{\circ}$ angle and $5 \mathrm{~cm}$ away from the ablated spot, to avoid saturation of the ultrasonic sensor while recording the laser-induced acoustic wave during plasma formation or the ablation process. Data were collected using LabVIEW (version 2016a) and information was extracted from the samples using MATLAB (version R2018b) software.

\section{Statistical Analysis}

Statistical analysis and calculations were performed in MATLAB software. We suppressed the phase shift of the measured ASW signal in the time domain by only using the amplitude spectrum determined using the fast Fourier transform at a sampling rate of $10 \mathrm{MHz}$. The average of two ASW spectra was calculated to improve the signal-to-noise ratio between each measured ASW amplitude spectrum. We split the amplitude spectrum into three equal frequency bands (low-, mid-, and highfrequency). Each frequency band was used as an input for the PCA. PCA was used to reduce the complexity of high-dimensional data by maintaining the same patterns and trends of the ASW field [50]. To classify tissue, we investigated the processed acoustics by looking at the amplitude-frequency band in which we achieved the best average classification error for each tissue type.

We compared the performance of PCA combined with either a quadratic and Gaussian SVMs or an ANN method. To implement the architecture of the SVM models, we used the fitcecoc function available in MATLAB (version R2018b) with the polynomial based kernel (with order of 4) and the gaussian kernel. For the ANN, we used the pattern network function combined with the Tan-Sigmoid activation function for hidden layers and Softmax activation function for the output layer. This is because multilayer networks can use the Tan-Sigmoid function and the output neurons are often used for pattern recognition problems. Furthermore, the Softmax function which was also known as a normalized

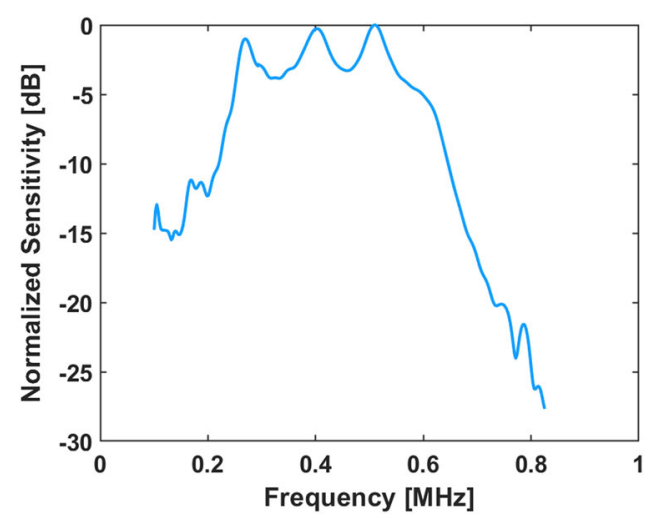

Fig. 3. Measured frequency response of the custom-made aircoupled piezoelectric transducer in reception mode. 
exponential function was employed to the layer's output to predict the label $[51,52]$. We evaluated the performance of our models fairly on a single computer with specification of $2.4 \mathrm{GHz}$ Intel Core i7 processor, $16 \mathrm{~GB} 1867 \mathrm{MHz}$ DDR3 memory.

During the training phase of the quadratic and Gaussian SVM, we used scores from the set of training data combined with a quadratic and Gaussian function kernel to set the boundary of the trained SVM. Testing scores within the boundary were considered as true positives or correct positive prediction and false positives (FP) also known as incorrect positive prediction otherwise. The criterion used by the two types of SVMs is based on margin maximization between the two data classes of tissues. The margin is the distance between the hyperplanes bounding in each class, wherein the hypothetical perfectly separable case, no observation may lie [53]. For the ANN method, we used one input layer, one hidden layer, and one output layer to build the network. The input layer was made of three neurons. The single hidden layer and the output layer were made of 10 neurons and one neuron, respectively. Then the Backpropagation algorithm was used to train the 10 neurons of the hidden layer using gradient descent. Similar to the SVM methods, the first three PCA scores from the set of data points were used as input sets of the ANN. A summary of the signal processing pipeline for tissue classification is given in Figure 4. For the SVMs, a set of 14,400 data points (or 7,200 averages of two spectra), measured from 10 craters in four proximal and distal femurs, was used as training data, while a set of 3,600 data points (or 1,800 averages of two spectra), measured from ten craters in the remaining proximal and distal femur, was considered as testing data for both lasers.

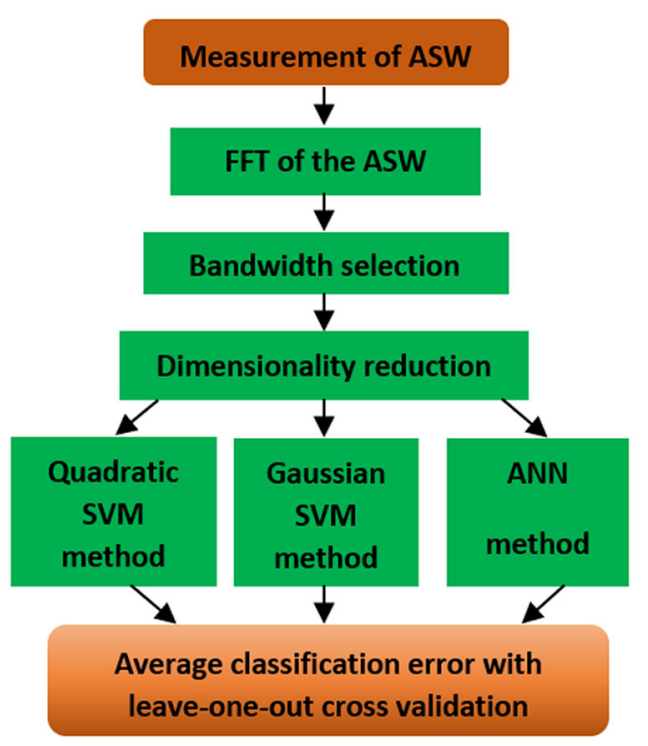

Fig. 4. Flowchart of the signal processing methods for tissue classification. ANN, artificial neural network; ASW, acoustic shock wave; FFT, fast Fourier transform; SVM, support vector machine.
We, then, simultaneously classified classes of tissues, using proximal and distal femur cross-validation. In the case of ANN, a set of 10,800 data points, measured from 10 craters in three proximal and distal femurs from the first three porcine, was used as training data. Then, a set of 3,600 data points, measured from 10 craters in one distal femur from the third porcine, was used as validation data. During the testing phase, a set of 3,600 data points, measured from 10 craters in the remaining proximal and distal femur from the fourth porcine, was considered as testing data for both lasers. The ground truth was obtained by ensuring that the tissue labels were correctly observed, and each tissue was uniformed. During the classification phase, the average error was calculated based on the mean error of five cross validated results from five folds-five proximal and distal femurs extracted from five different porcines. From the confusion matrix, percentage errors rate in the testing-data-based scores from each specimen were calculated as the number of all incorrect predictions divided by the total number of the dataset. The worst error rate is 0 (or $0 \%$ ), whereas the best is 1 (or 100\%) (Equation 1)

$$
\% \mathrm{ER}=\frac{\mathrm{FP}+\mathrm{FN}}{\text { Total }_{\text {dataset }}} \times 100
$$

where $\mathrm{FN}$ is the false negative or incorrect negative prediction and FP, false positive or incorrect positive prediction.

\section{RESULTS}

The acoustic signals in the time domain were acquired during laser ablation for hard bone, soft bone, muscle, fat, and skin using (Fig. 2a) ns-Nd:YAG and (Fig. 2b) $\mu$ sEr:YAG lasers. By comparing the ASWs generated by the different tissues, we found that the peak-to-peak amplitude of the ASWs generated by the hard bone specimen and measured by the ACT were higher than those generated by the surrounding tissues (soft bone, muscle, fat, and skin). The peak-to-peak value of the ASWs generated for each tissue with the Nd:YAG laser was $\sim 7$ times higher than those generated with the Er:YAG laser. In addition, the acoustic signal duration (wt) generated by the $\mathrm{Nd}$ :YAG laser ( $\mathrm{wt}=0.82$ milliseconds) was longer compared with the one generated by the Er:YAG laser (wt = 0.70 milliseconds). The corresponding frequency domain of the ASWs for each tissue is depicted in Figure 5a and b. The amplitude spectrum of hard bone is higher compared with that of the surrounding tissues (Fig. 5a and b). We split the spectrum into three equal frequency bands, as shown in Figure 5a and b. The data suggest at low and mid-frequency between $0.115-0.27$ and $0.27-0.53 \mathrm{MHz}$, the classification of hard bone from the surrounding tissues was more accurate than in other bands when ablating with Er:YAG and Nd:YAG, respectively (Table 2). The classification of tissue types based on the analysis of the measured ASWs is depicted in Figures 6. The features that were chosen for PCA show $96.10 \%$ and $97.50 \%$ of the total variance in the acoustic waves generated with the 

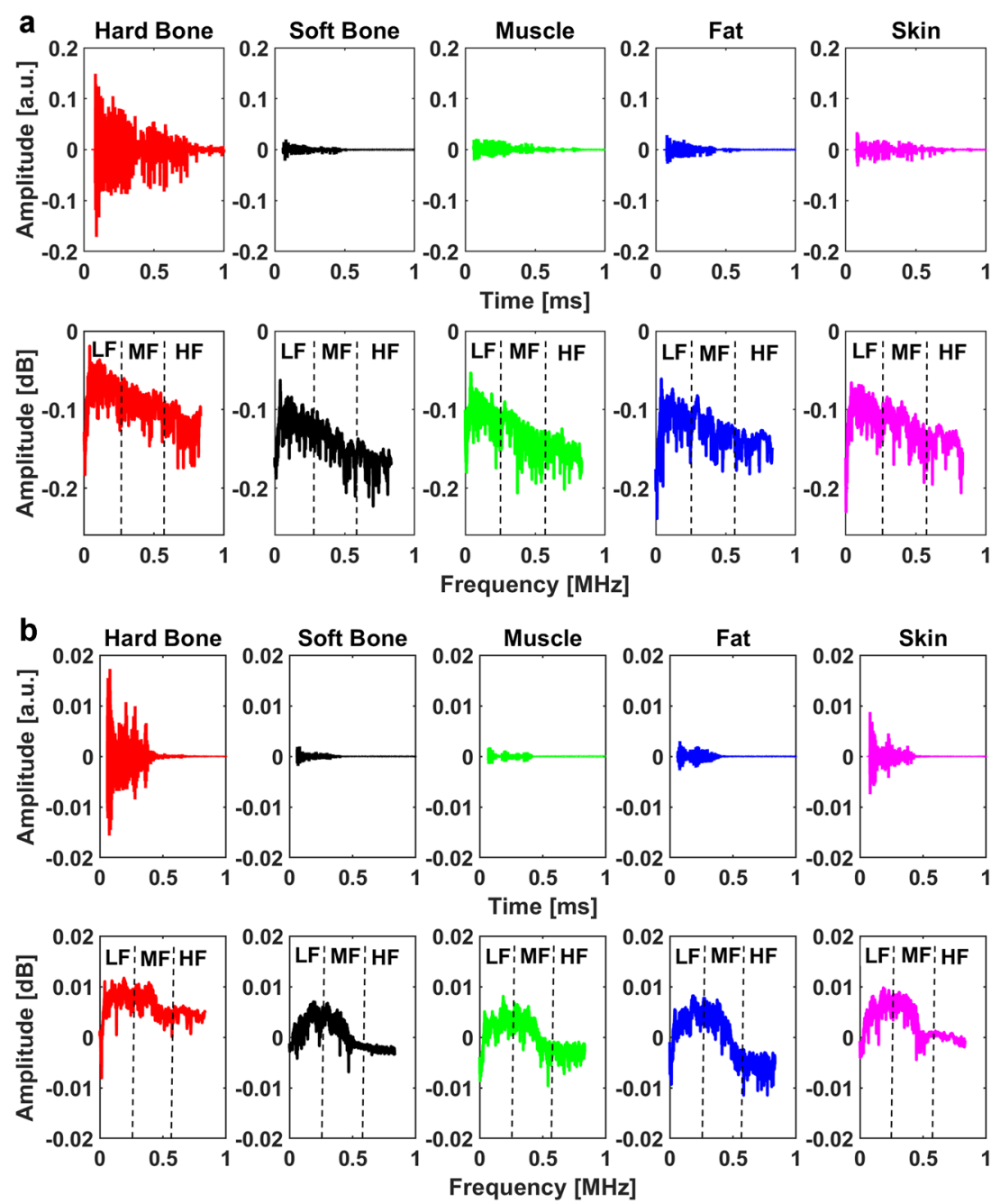

Fig. 5. (a) Measured shock wave using ns-Nd:YAG laser with time (Top row) and frequency domain (Bottom row): low-frequency (LF), mid-frequency (MF), high -frequency (HF). (b) Measured acoustic wave using $\mu \mathrm{s}$-Er:YAG laser with time (Top row) and frequency domain (Bottom row): LF, MF, HF. Er:YAG, erbium-doped yttrium aluminum garnet; Nd:YAG, neodimium-doped yttrium aluminum garnet.

Nd:YAG laser and the Er:YAG laser, respectively. We observed a better classification performance with the ANN than with the quadratic and Gaussian SVM methods, for both lasers-at low and mid-frequency for Er:YAG and Nd:YAG, respectively. Table 2 further shows that the ANN performed the best, with an average classification error for all tissues of $5.01 \pm 5.06 \%$ and $9.12 \pm 3.39 \%$, using the Nd:YAG and Er:YAG lasers, respectively. Then, the Gaussian-SVM performed better than the quadratic SVM during the ablation with both lasers. The Gaussian-SVM yielded average classification errors of $15.17 \pm 13.12 \%$ and $16.85 \pm 7.59 \%$, using the Nd:YAG and Er:YAG lasers, respectively (Table 2). The worst performance was achieved with the quadratic-SVM with a classification error of $50.34 \pm 35.04 \%$ and $69.96 \pm 25.49 \%$, using the Nd:YAG and Er:YAG lasers. Average classification errors with leave-one-out cross validation for ANN and SVMs are detailed in Tables $3-5$. The computational time for testing the ANN and SVM based model is in the order of 11-20 milliseconds. Summary of each computational time is in Table 6.

\section{DISCUSSION}

We observed greater amplitudes for the ASWs generated with the Nd:YAG laser than the ones generated by the Er:YAG laser (Fig. 5a and b). This amplitude value suggests that the generation of acoustic waves is more efficient with the nanosecond Nd:YAG laser. This is because the amplitude of the generated ASWs depends not only energy but also pulse duration, focusing conditions, and mainly tissue type being ablated. In fact, at the same laser pulse duration and focusing conditions, the acoustic amplitude increases with energy [54,55]. Therefore, if both lasers have the same pulse duration, the acoustic amplitude from the Er:YAG laser at $940 \mathrm{~mJ}$, should be 

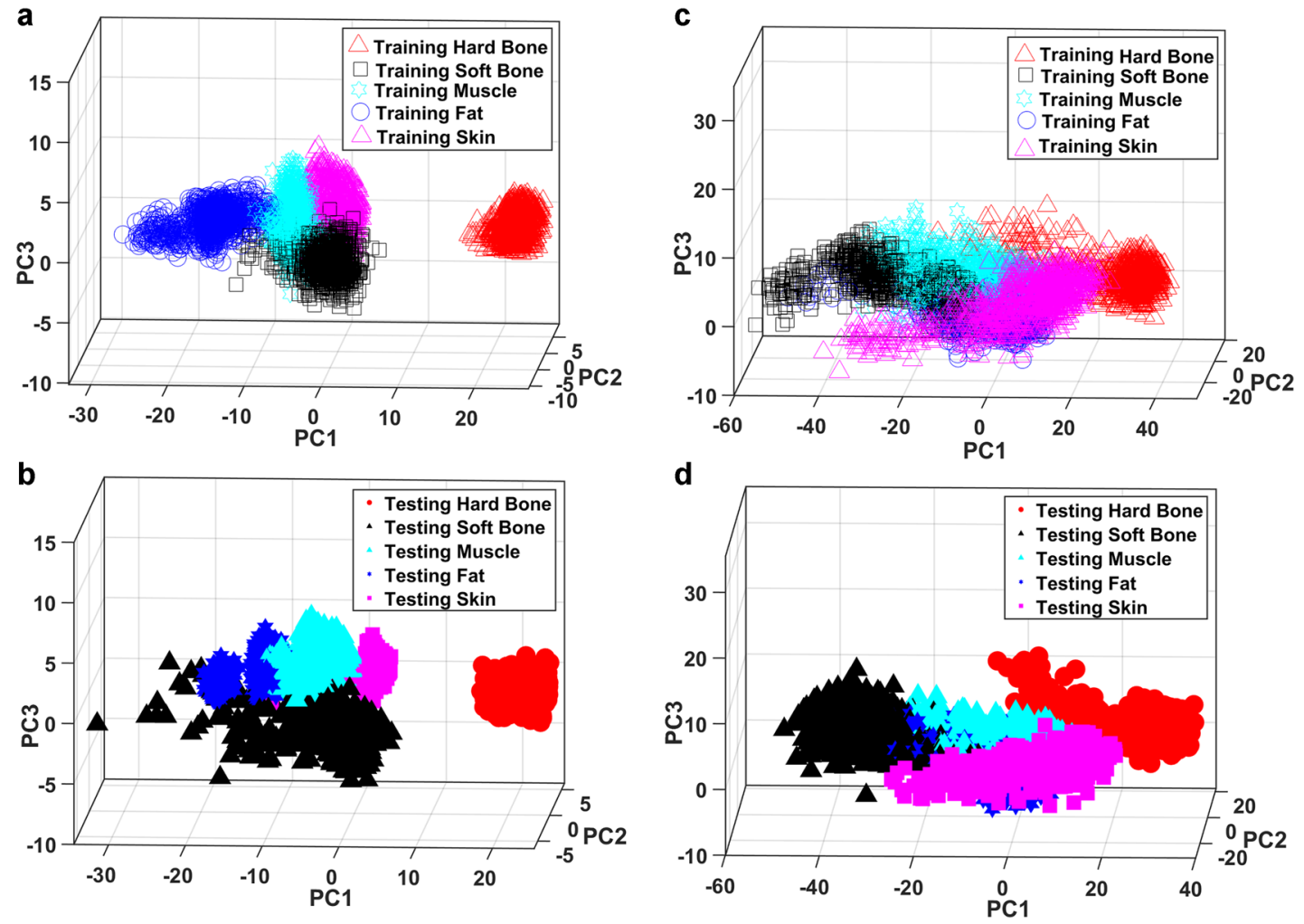

Fig. 6. Seven thousand and two hundred scores from training data (a and $\mathbf{c}$; using Nd:YAG laser and Er:YAG laser, respectively) and classification of 1,800 scores from testing data (b and d; using Nd:YAG laser and Er:YAG laser, respectively) based on margin maximization between the five data classes of the training data for hard and soft bone, muscle, fat, and skin. Er:YAG, erbiumdoped yttrium aluminum garnet; Nd:YAG, neodimium-doped yttrium aluminum garnet.

higher than that of with the Nd:YAG laser at $200 \mathrm{~mJ}$. However, in this experimental study, both lasers operate at different pulse durations. Therefore, even though the $\mathrm{Nd}$ :YAG has less pulse energy than the Er:YAG, we observe greater amplitudes for the ASWs generated with the Nd:YAG laser than the ones generated by the Er:YAG laser (Fig. 5a and b). The Nd:YAG generated acoustic wave is much more effective, generating high frequencies, while Er:YAG is not only less efficient (smaller amplitude) but also more frequency selective, being more efficient in the band $0.1-0.4 \mathrm{MHz}$. This information could be used to adapt and optimize receiver transducers for this purpose. The higher values were theoretically expected, as the ablation with the Nd:YAG is based on plasma mediation, which increases the pressure energy measured by the transducer. The Er:YAG laser source results in thermal ablation, thus, most of the light energy is absorbed by the exposed tissue and transformed into heat $[12,20,56]$.

This is likely also the reason the acoustic signal duration generated by the Nd:YAG laser was longer as compared to the one generated by the Er:YAG laser (Fig. 5a and c). This behavior (acoustic signal duration) suggests a more resonant ASW generation while using the Nd:YAG laser. The bandwidth of the ASW's response is broad because the measured acoustic waves result from transient signals. For Er:YAG, we used the low-frequency band of $0.115-0.27 \mathrm{MHz}$ (Fig. 5d) to classify tissue types because thermal ablation generates acoustic waves (not shock wave and acoustic wave is always in the low-frequency range); and important parameters of acoustic waves lie in this region. Moreover, the transducer is fully characterized at $0.1-0.8 \mathrm{MHz}$ (Fig. 4), by focusing the analysis

TABLE 1. A Summarizing Table of Both Laser's Parameters for all Five Tissues

\begin{tabular}{lcccccc}
\hline Laser types & $\begin{array}{c}\text { Pulse } \\
\text { energy }(\mathrm{mJ})\end{array}$ & $\begin{array}{c}\text { Pulse } \\
\text { duration }(\mu \mathrm{s})\end{array}$ & $\begin{array}{c}\text { Repetition } \\
\text { rate }(\mathrm{Hz})\end{array}$ & $\begin{array}{c}\text { Laser } \\
\text { wavelength }(\mathrm{nm})\end{array}$ & $\begin{array}{c}\text { Distance between } \\
\text { lens and tissue }(\mathrm{mm})\end{array}$ & $\begin{array}{c}\text { Water flow } \\
\text { rate }(\mathrm{ml} / \mathrm{s})\end{array}$ \\
\hline Nd:YAG & 200 & $5 \times 10^{-3}$ & 2 & 532 & 30 & 0.1 \\
Er:YAG & 940 & 400 & 2 & 2940 & 30 & 0.1
\end{tabular}

Er:YAG, erbium-doped yttrium aluminum garnet; Nd:YAG, neodimium-doped yttrium aluminum garnet. 
TABLE 2. Average Error of All Tissues for Both Lasers Using SVM and ANN at Low, Mid, and High-Frequency for Both Nd:YAG and the Er:YAG Ablation

\begin{tabular}{cccc}
\hline \multicolumn{4}{c}{ Comparison of three methods } \\
$\begin{array}{c}\text { Quadratic- } \\
\text { SVM }\end{array}$ & $\begin{array}{c}\text { Gaussian- } \\
\text { SVM }\end{array}$ & ANN \\
Laser types & $55.89 \%$ & $18.37 \%$ & $8.81 \%$ \\
\hline Low-frequency: $0.115-0.27 \mathrm{MHz}$ & & \\
Nd:YAG & $69.96 \%$ & $16.85 \%$ & $9.12 \%$ \\
Er:YAG & $50.34 \%$ & $15.17 \%$ & $5.01 \%$ \\
Mid-frequency: $0.27-0.53 \mathrm{MHz}$ & $30.82 \%$ & $14.62 \%$ \\
Nd:YAG & $72.4 \%$ & & \\
Er:YAG & $53.39 \%$ & $20.04 \%$ & $10.48 \%$ \\
High-frequency: & $0.53-0.80 \mathrm{MHz}$ & $26.66 \%$ & $21.15 \%$ \\
Er:YAG & $76.52 \%$ & &
\end{tabular}

ANN, artificial neural network; Er:YAG, erbium-doped yttrium aluminum garnet; Nd:YAG, neodimium-doped yttrium aluminum garnet; SVM, support vector machine.

above $0.1 \mathrm{MHz}$, we were always able to reduce this unwanted signal component, thereby improving the tissue classification. Confusion matrices indicate that tissue classification has the highest accuracy within this band as highlighted in Table 1. In contrast to the Er:YAG, cutting tissue with Nd:YAG is based on the plasma mediated ablation and emits the shock waves in which spectra extend beyond $1 \mathrm{MHz}$ [57]. However, the $-3 \mathrm{~dB}$ bandwidth of our commercial microphones do not typically exceed $0.9 \mathrm{MHz}$ and the resonant frequency is at $0.4 \mathrm{MHz}$.
Therefore, using the mid-frequency band $0.27-0.53 \mathrm{MHz}$ (Fig. 5b), the confusion matrices indicate that the tissue classification has the highest accuracy within this band. Another advantage of using this region is it overlaps with the frequency band where the transducer has highest sensitivity.

Hard bone specimens generated higher peak-to-peak ASW values than the soft tissues did because soft tissues contain $79 \%$ water, while hard bone is made up of $85-95 \%$ carbonated hydroxyapatite [58]. Thus, we believe that the carbonated hydroxyapatite resulted in a higher amplitude due to its compact structure $[10,58,59]$. On the basis of this compact structure (physical propriety) of hard bone, it was possible to classify hard bone against soft tissues, during both lasers (details are in Table 3 and 4). In general soft tissues - except the classification error for muscle tissue using the Nd:YAG-had the highest average classification errors compared with hard bone. This is probably due to the structure of the soft tissues, which consist of fatty connecting tissue and contain more water than bone, resulting in lower amplitude ASWs as compared with bone.

The ANN method showed a superior classification performance as compared to the quadratic- and GaussianSVM methods; the Gaussian-SVM performed better than the quadratic-SVM method (Tables 2-5). One explanation for this result is that we trained our classifier using a large amount of data; with more data, the ANN and Gaussian kernel perform generally better than the polynomial kernels. Therefore, using the ANN and Gaussian kernel, we can model more functions within its function space than using the polynomial kernels. However, if we

TABLE 3. Confusion Matrix for Hard Bone, Soft Bone, Fat, Skin, and Muscle Tissue at Low- and Mid-Frequency During Nd:YAG and the Er:YAG Ablation, Respectively

\begin{tabular}{|c|c|c|c|c|c|c|}
\hline \multicolumn{7}{|c|}{ Quadratic SVM method } \\
\hline \multirow[b]{2}{*}{ Tissue } & \multicolumn{3}{|c|}{ Classified as } & \multirow[b]{2}{*}{ Muscle } & \multirow[b]{2}{*}{ Skin } & \multirow{2}{*}{$\begin{array}{c}\text { Average } \\
\text { classification error }\end{array}$} \\
\hline & Hard bone & Soft bone & Fat & & & \\
\hline \multicolumn{7}{|l|}{ Hard bone } \\
\hline Nd:YAG & 1645 & 0 & 0 & 139 & 16 & $8.60 \%$ \\
\hline Er:YAG & 1464 & 16 & 63 & 175 & 82 & $18.70 \%$ \\
\hline \multicolumn{7}{|l|}{ Soft bone } \\
\hline Nd:YAG & 140 & 449 & 1209 & 1 & 1 & $75.10 \%$ \\
\hline Er:YAG & 637 & 265 & 137 & 278 & 483 & $85.30 \%$ \\
\hline \multicolumn{7}{|l|}{ Fat } \\
\hline Nd:YAG & 263 & 310 & 609 & 0 & 618 & $66.20 \%$ \\
\hline Er:YAG & 643 & 36 & 194 & 319 & 638 & $89.22 \%$ \\
\hline \multicolumn{7}{|l|}{ Muscle } \\
\hline Nd:YAG & 109 & 0 & 0 & 1667 & 24 & $7.40 \%$ \\
\hline Er:YAG & 717 & 132 & 63 & 429 & 459 & $76.17 \%$ \\
\hline \multicolumn{7}{|l|}{ Skin } \\
\hline Nd:YAG & 2 & 1342 & 356 & 0 & 100 & $94.40 \%$ \\
\hline Er:YAG & 1045 & 9 & 41 & 353 & 352 & $80.40 \%$ \\
\hline
\end{tabular}

Numbers in the table are testing-data-based scores from each specimen.

Er:YAG, erbium-doped yttrium aluminum garnet; Nd:YAG, neodimium-doped yttrium aluminum garnet; SVM, support vector machine. 
TABLE 4. Confusion Matrix for Hard Bone, Soft Bone, Fat, Skin, and Muscle Tissue at Low- and Mid-Frequency During Nd:YAG and the Er:YAG Ablation, Respectively

\begin{tabular}{|c|c|c|c|c|c|c|}
\hline \multicolumn{7}{|c|}{ Gaussian SVM method } \\
\hline \multirow[b]{2}{*}{ Tissue } & \multicolumn{3}{|c|}{ Classified as } & \multirow[b]{2}{*}{ Muscle } & \multirow[b]{2}{*}{ Skin } & \multirow{2}{*}{$\begin{array}{c}\text { Average } \\
\text { classification error }\end{array}$} \\
\hline & Hard bone & Soft bone & Fat & & & \\
\hline \multicolumn{7}{|l|}{ Hard bone } \\
\hline Nd:YAG & 1682 & 0 & 0 & 118 & 0 & $6.55 \%$ \\
\hline Er:YAG & 1718 & 4 & 0 & 0 & 78 & $4.56 \%$ \\
\hline \multicolumn{7}{|l|}{ Soft bone } \\
\hline Nd:YAG & 0 & 1669 & 46 & 9 & 76 & $7.28 \%$ \\
\hline Er:YAG & 3 & 1579 & 3 & 30 & 185 & $12.30 \%$ \\
\hline \multicolumn{7}{|l|}{ Fat } \\
\hline Nd:YAG & 0 & 273 & 1322 & 29 & 176 & $26.60 \%$ \\
\hline Er:YAG & 0 & 25 & 1390 & 227 & 159 & $22.83 \%$ \\
\hline \multicolumn{7}{|l|}{ Muscle } \\
\hline Nd:YAG & 0 & 3 & 0 & 1797 & 0 & $0.17 \%$ \\
\hline Er:YAG & 0 & 63 & 194 & 1473 & 70 & $18.17 \%$ \\
\hline \multicolumn{7}{|l|}{ Skin } \\
\hline $\mathrm{Nd}: \mathrm{YAG}$ & 0 & 171 & 249 & 215 & 1165 & $35.27 \%$ \\
\hline Er:YAG & 105 & 159 & 107 & 105 & 1324 & $26.4 \%$ \\
\hline
\end{tabular}

Numbers in the table are testing-data-based scores from each specimen.

Er:YAG, erbium-doped yttrium aluminum garnet; Nd:YAG, neodimium-doped yttrium aluminum garnet; SVM, support vector machine.

TABLE 5. Confusion Matrix for Hard Bone, Soft Bone, Fat, Skin, and Muscle Tissue at Low- and Mid-Frequency During Nd:YAG and the Er:YAG Ablation, Respectively

ANN method

\begin{tabular}{|c|c|c|c|c|c|c|}
\hline \multirow[b]{2}{*}{ Tissue } & \multicolumn{3}{|c|}{ Classified as } & \multirow[b]{2}{*}{ Muscle } & \multirow[b]{2}{*}{ Skin } & \multirow{2}{*}{$\begin{array}{c}\text { Average classification } \\
\text { error }\end{array}$} \\
\hline & Hard bone & Soft bone & Fat & & & \\
\hline \multicolumn{7}{|l|}{ Hard bone } \\
\hline Nd:YAG & 1800 & 0 & 0 & 0 & 0 & $0 \%$ \\
\hline Er:YAG & 1750 & 0 & 0 & 0 & 50 & $2.78 \%$ \\
\hline \multicolumn{7}{|l|}{ Soft bone } \\
\hline Nd:YAG & 0 & 1691 & 41 & 1 & 67 & $6.06 \%$ \\
\hline Er:YAG & 0 & 1653 & 8 & 59 & 80 & $8.17 \%$ \\
\hline \multicolumn{7}{|l|}{ Fat } \\
\hline Nd:YAG & 0 & 44 & 1722 & 0 & 33 & $4.28 \%$ \\
\hline Er:YAG & 0 & 109 & 1594 & 24 & 73 & $11.44 \%$ \\
\hline \multicolumn{7}{|l|}{ Muscle } \\
\hline Nd:YAG & 0 & 7 & 1 & 1792 & 0 & $0.44 \%$ \\
\hline Er:YAG & 0 & 51 & 64 & 1603 & 82 & $10.94 \%$ \\
\hline \multicolumn{7}{|l|}{ Skin } \\
\hline Nd:YAG & 0 & 49 & 208 & 0 & 1543 & $14.28 \%$ \\
\hline Er:YAG & 24 & 99 & 82 & 16 & 1579 & $12.28 \%$ \\
\hline
\end{tabular}

Numbers in the table are testing-data-based scores from each specimen.

Er:YAG, erbium-doped yttrium aluminum garnet; Nd:YAG, neodimium-doped yttrium aluminum garnet; SVM, support vector machine. 
TABLE 6. Average Computational Time to the Test of the SVM and ANN Models for Both Lasers

\begin{tabular}{lccc}
\hline $\begin{array}{l}\text { Laser } \\
\text { types }\end{array}$ & $\begin{array}{c}\text { Quadratic- } \\
\text { SVM (ms) }\end{array}$ & $\begin{array}{c}\text { Gaussian- } \\
\text { SVM (ms) }\end{array}$ & $\begin{array}{c}\text { ANN } \\
(\mathrm{ms})\end{array}$ \\
\hline Nd:YAG & 20.02 & 13.08 & 11.09 \\
Er:YAG & 23.14 & 14.12 & 11.17 \\
\hline
\end{tabular}

ANN, artificial neural network; Er:YAG, erbium-doped yttrium aluminum garnet; Nd:YAG, neodimium-doped yttrium aluminum garnet; SVM, support vector machine.

had used fewer data, then, a polynomial kernel would have been a much better fit to the measured data than the gradient and Gaussian. In fact, the ANN and Gaussian SVM method are nonparametric methods, meaning that the complexity of the model is potentially infinite; its complexity can grow with the data [53,60]. More data will be able to represent more and more complex relationshipshowever, this simple classification approach also hase limits as it has a single hidden layer. In contrast, the quadraticSVM methods have a fixed size (parametric model), so after a certain point, the model will be saturated, and increasing the data would not improve the classifier. In this case, we had a large amount of data and very weak assumptions about the challenge, thus a nonparametric method served us better.

Furthermore, we used leave-one-out cross validation because, in general, there is a tradeoff between accuracy and generalization. The more accurate the classifier is with the training data, the less likely it is to generalize (though it depends on the training data). Furthermore, we used PCA to reduce the data dimensionally at each frequency range. This is because principal components (PCs) consecutively maximize variance and can be obtained from the eigenvalues/eigenvectors of a covariance matrix [61]. When all variables are measured in the same units, covariance-based PCA may be suitable. In general, the first PCs are dominated by high-variance variables and mostly represent the variance of each data. Therefore, by confining the number of eigenvalues and eigenvectors to the first three PCs, we aim to keep the most represent variance of each data and improve the speed of online classifier when transferring feedback sensor to other systems for in vivo measurements.

In case that the online classifier produces more error, more PCs can be used as a solution even if the computational time increases. In addition, the angle between the transducer and the ablated spot must be adjusted to $45^{\circ}$. Moreover, the transducer should be placed $5 \mathrm{~cm}$ away from the ablated spot to maintain the same condition as in this experimental setup. In case that the machine structure does not allow to fix the sensor position at the mentioned angle and distance, an alternative approach is to fix an omnidirectional transducer at any angle and distance in the linear regime to collect the data. Then, the data need to be normalized in the time domain to avoid the negative impact of the distance between the transducer and the ablated spot (because the amplitude value of the acoustic wave exponentially decays with the distance). To improve the machine learning approach, the system must be trained with a lot of data using femurs from different patients and body parts such as the skull. This is because depending on age, nutrition, and body parts of each patient, it is possible that the tissue response varies slightly. By making the laser cut other body parts such as skull or limb, the laser should be able to rotate around them while cutting. This requires an XYZ machine (controlled rotating holder). Laser combined with a robotic arm or endoscope to be able to control the movements is the next plan of MIRACLE project.

Furthermore, the feedback system must be also regularly trained when transferring results to another system or machine (new condition). This is because the sensor system could have different transfer functions or irrigation conditions, which can affect the raw data of acoustic measured time. Additionally, during ablation by the Er:YAG laser, carbonization can possibly appear at the ablated spot if the water cooling system is not appropriately set. To avoid this, automatic detection for early carbonization (dry tissue vs. wet tissue) must also be integrated in the current feedback to classify the ablated tissue and monitor early carbonization simultaneously in real time. As soon as the feedback system detects early carbonization, water flow rate of the cooling system will be increased to prevent carbonization. Currently, we used distilled water in ablation experiments to prevent earlier carbonization, distilled water can be replaced by physiological water which is more suitable for surgery. We have tested physiological water in our previous experiments in which we got a similar behavior to the distilled water. So, we continued using distilled water because it is already in our laboratory. When using the laser system in the clinic, we will perform experiments with the physiological water.

Moreover, in our previous work, we investigated optimum laser parameters for a long-pulsed high-energy laser to produce craters with minimal thermal damage under wet condition using an optical coherence tomography or confocal microscope, and a scanning electron microscope (SEM) [12]. The pulse duration and pulse energies were $0.5-10$ milliseconds and $0.75-15 \mathrm{~J}$, respectively. The confocal microscope was used for calculating the ablation efficiency but SEM was needed for analyzing craters of various morphologies to observe random charring, thermal damage, and cracks of hard and soft tissues. We planned to use the same method to investigate the difference in ablation and burning depth of hard tissue compared with soft tissue, using both lasers. Future work will also include a histological study in a cross-section for each tissue after the laser ablation, to fully evaluate the potential of the technique in terms of the reduction of bone damage compared with other techniques. For bone cutting, we applied fixed high energy pulses to produce ablation. That is why in our case, the light dose is always the same as the laser is used for bone ablation application. Hence, it is not needed to perform a classification with unseen data (at different laser energies) to conclude on the actual utility of the proposed 
scheme in realistic situations that might arise in the clinic. In addition, the time for cutting a real bone with laser is longer than when using standard mechanical tools such as a saw; however, the laser has more advantagesincluding functional cuts, contactless interaction, and faster wound healing-which gives an overall better outcome than mechanical surgery. The ablation rate at the surface $(\sim 1 \mathrm{~mm}$ depth$)$ is around 10 and $0.1 \mathrm{~mm} / \mathrm{s}$ for Er:YAG and Nd:YAG, respectively. However, at deeper ablation ( $10 \mathrm{~mm}$ depth) is $0.2 \mathrm{~mm} / \mathrm{s}$ for Er:YAG; we have not yet done with Nd:YAG for hole ablation. Additionally, in terms of contamination, for all the debris, we use the extraction system, or we just blow off all the debris using pressurized air. In contrast to the standard mechanical tools, laser ablation produces microparticles, and the bone is completely disintegrated.

Currently, the error rate is less than $10 \%$. This promising result led us to investigate advanced precision in signal classification to further reduce the classification error. To reach efficiency in processing, we plan to involve a cutting-edge deep learning technique such as a onedimensional Convolutional Neural Network to classify these ASWs. We also plan to filter out misclassified data based on the temporal discrimination of tissue typesthat is, when doing a line cut on top of hard bone with laser, we detect hard bone during the last ten shots and suddenly, we detect skin in one shot followed by the detection of hard bone again, the software will just filter out this misclassified skin and considers it as outlier or hard bone. Additionally, we are also investigating sensitive sensors such as optical sensors to improve ASW measurement. We believe that with better data measured, classifiers will better detect tissues types compared to when using the ACT.

\section{CONCLUSION}

Our aim was to simultaneously classify tissue types during laser ablation by measuring the ASWs generated with an ACT and by processing the information using three different machine learning methods. The ANN, quadratic or Gaussian-SVM-methods were combined with PCA during classification. In the experiments, we used two different lasers to ablate tissue types and to generate ASWs. The peak-to-peak amplitudes of the ASW generated by the hard bone specimen and measured by the ACT were consistently higher than those generated by the surrounding tissues (soft bone, muscle, fat, and skin). On the basis of the average error for all tissues using both lasers, the ANN performed best in terms of classifying all tissues during ablation. Thus, the measured ASWs can be quantified and used to control the laser cutting process in a feedback control loop. By classifying tissue type during ablation, we avoid damaging an important tissue such as bone marrow (or soft bone). As soon the signal of the ASWs is classified as soft bone, the laser will stop ablating (the stopping point).

Future work includes the development of optical sensors to detect the ASW fields. Optical techniques are very sensitive to sharp changes in pressure with wide bandwidths compared with commercially-available transducers [57].

\section{ACKNOWLEDGMENTS}

This project is part of the MIRACLE (short for Minimally Invasive Robot-Assisted Computer-guided LaserosteotomE) project funded by the Werner Siemens Foundation. The ACTs part was funded by (DPI2016-78876-R-AEI/FEDER, UE) from the Spanish State Research Agency (AEI) and the European Regional Development Fund (ERDF/FEDER).

\section{REFERENCES}

1. Beltrán L, Abbasi H, Rauter G, Friederich N, Cattin P, Zam A. Effect of laser pulse duration on ablation efficiency of hard bone in microseconds regime. In: Third International Conference on Applications of Optics and Photonics. International Society for Optics and Photonics: Vol 10453. 2017. p 104531S.

2. Abbasi H, Beltrán L, Rauter G, Guzman R, Cattin PC, Zam A. Effect of cooling water on ablation in Er:YAG laserosteotome of hard bone. In: Third International Conference on Applications of Optics and Photonics. International Society for Optics and Photonics: Vol 10453. 2017. p 104531I.

3. Romeo U, Del Vecchio A, Palata G, Tenore G, Visca P, Maggiore C. Bone damage induced by different cutting instruments: an in vitro study. Brazilian Dent J 2009;20:162-168. http://www.scielo.br/scielo.php?script=sci_arttext\&pid= S0103-64402009000200013\&nrm=iso

4. Baek K-W, Deibel W, Marinov D, et al. A comparative investigation of bone surface after cutting with mechanical tools and Er:YAG laser. Lasers Surg Med 2015;47(5): 426-432. https://doi.org/10.1002/lsm.22352

5. Abbasi H, Rauter G, Guzman R, Cattin PC, Zam A. Differentiation of femur bone from surrounding soft tissue using laser-induced breakdown spectroscopy as a feedback system for smart laserosteotomy (SPIE Photonics Europe). SPIE 2018. 60.

6. Peng Q, Juzeniene A, Chen J, et al. Lasers in medicine. Rep Prog Phys 2008;71(5):056701.

7. Peavy GM, Reinisch L, Payne JT, Venugopalan V. Comparison of cortical bone ablations by using infrared laser wavelengths 2.9 to $9.2 \mu \mathrm{m}$. Lasers Surg Med 1999;25(5):421-434.

8. Walsh JT, Jr, Deutsch TF. Er:YAG laser ablation of tissue: measurement of ablation rates. Lasers Surg Med 1989;9(4):327-337.

9. Kuscer L, Diaci J. Measurements of erbium laser-ablation efficiency in hard dental tissues under different water cooling conditions. SPIE 2013;18:11.

10. Kang HW, Rizoiu I, Welch AJ. Hard tissue ablation with a spray-assisted mid-IR laser. Phys Med Biol 2007;52(24):7243-7259. http://stacks.iop.org/0031-9155/ $52 / \mathrm{i}=24 / \mathrm{a}=004$

11. Li ZZ, Reinisch L, Van de Merwe WP. Bone ablation with Er:YAG and CO2 laser: Study of thermal and acoustic effects. Lasers Surg Med 1992;12(1):79-85.

12. Nguendon Kenhagho K, Shevchik S, Saeidi F, et al. Characterization of ablated bone and muscle for long-pulsed laser ablation in dry and wet conditions. Materials 2019;12(8):1338. http://www.mdpi.com/1996-1944/12/8/1338

13. Jowett N, Wöllmer W, Reimer R, et al. Bone ablation without thermal or acoustic mechanical injury via a novel picosecond infrared laser (PIRL). Otolaryngol Head Neck Surg 2014;150(3):385-393.

14. Ana PA, Bachmann L, Zezell DM. Lasers effects on enamel for caries prevention. Laser Phys 2006;16(5):865-875. https:// doi.org/10.1134/s1054660x06050197

15. Abbasi H, Rauter G, Guzman R, Cattin PC, Zam A. Laserinduced breakdown spectroscopy as a potential tool for autocarbonization detection in laserosteotomy. J Biomed Opt 2018;23(7):071206. 
16. Lewandrowski K-U, Lorente C, Schomacker KT, Fiotte TJ, Wilkes JW, Deutsch TF. Use of the Er:YAG laser for improved plating in maxillofacial surgery: Comparison of bone healing in laser and drill osteotomies. Lasers Surg Med 1996;19(1):40-45. https://doi.org/10.1002/(sici)1096-9101(1996)19:1<40::aidlsm6 $>3.0 . c 0 ; 2-q$

17. Fried D, Ashouri N, Breunig T, Shori R. Mechanism of water augmentation during IR laser ablation of dental enamel. Lasers Surg Med 2002;31(3):186-193.

18. Tulea C-A, Caron J, Gehlich N, Lenenbach A, Noll R, Loosen P. Laser cutting of bone tissue under bulk water with a pulsed ps-laser at $532 \mathrm{~nm}$. SPIE 2015;20:9.

19. Abbasi H, Rauter G, Guzman R, Cattin PC, Zam A. Plasma plume expansion dynamics in nanosecond Nd: YAG laserosteotome. In: High-Speed Biomedical Imaging and Spectroscopy III. International Society for Optics and Photonics: Vol 10505. 2018. p 1050513.

20. Kenhagho HN, Rauter G, Guzman R, Cattin PC, Zam A. Comparison of acoustic shock waves generated by micro and nanosecond lasers for a smart laser surgery system. SPIE BiOS 2018;10484:9.

21. Jacques SL. Role of tissue optics and pulse duration on tissue effects during high-power laser irradiation. Appl Opt 1993;32(13):2447-2454.

22. Bay E, Deán-Ben XL, Pang GA, Douplik A, Razansky D. Real-time monitoring of incision profile during laser surgery using shock wave detection. J Biophotonics 2015;8(12):102-111. https://doi.org/10.1002/jbio.201300151

23. Oraevsky AA, Jacques SL, Tittel FK. Mechanism of laser ablation for aqueous media irradiated under confined-stress conditions. J Appl Phys 1995;78(2):1281-1290.

24. Oraevsky AA, Jacques SL, Esenaliev RO, Tittel FK. Pulsed laser ablation of soft tissues, gels, and aqueous solutions at temperatures below $100^{\circ} \mathrm{C}$. Lasers Surg Med 1996;18(3):231-240. https://doi.org/10.1002/(sici) 1096-9101(1996)18:3<231::aid-lsm3>3.0.co;2-t

25. Nguendon HK, Faivre N, Meylan B, et al. Characterization of ablated porcine bone and muscle using laser-induced acoustic wave method for tissue differentiation. In: European Conferences on Biomedical Optics. SPIE: Vol 10417. 2017. p 10.

26. Nguendon HK, Faivre N, Meylan B, et al. Characterization of ablated porcine bone and muscle using laser-induced acoustic wave method for tissue differentiation. In: European Conference on Biomedical Optics. Optical Society of America. 2017. p 104170N.

27. Brecht H-PF, Su R, Fronheiser MP, Ermilov SA, Conjusteau A, Oraevsky AA. Whole-body three-dimensional optoacoustic tomography system for small animals. J Biomed Opt 2009;14(6):1-8. https://doi.org/10.1117/1.3259361. 8 [Online]. Available.

28. Fehm TF, Deán-Ben XL, Razansky D. Four dimensional hybrid ultrasound and optoacoustic imaging via passive element optical excitation in a hand-held probe. Appl Phys Lett 2014;105(17):173505.

29. Deán-Ben XL, Pang GA, Montero de Espinosa F, Razansky D. Non-contact optoacoustic imaging with focused aircoupled transducers. Appl Phys Lett 2015;107(5):051105.

30. Michau S, Mauchamp P, Dufait R. Piezocomposite 30MHz linear array for medical imaging: Design challenges and performances evaluation of a 128 elements array. In: IEEE Ultrasonics Symposium, 2004. IEEE: Vol 2. 2004. pp 898-901.

31. Franjic K, Cowan ML, Kraemer D, Miller RJD. Laser selective cutting of biological tissues by impulsive heat deposition through ultrafast vibrational excitations. Opt Express 2009;17(25):22937-22959. https://doi.org/10.1364/ OE.17.022937. 12/07 2009.

32. Landa FJO, Özsoy C, Deán-Ben XL, Razansky D. Optoacoustic monitoring of RF ablation lesion progression (SPIE BiOS). SPIE 2019;94:117-123.

33. Rebling J, Landa FJO, Deán-Ben XL, Douplik A, Razansky D. Integrated catheter for simultaneous radio frequency ablation and optoacoustic monitoring of lesion progression. Opt Lett 2018;43(8):1886-1889.

34. Landa FJO, Deán-Ben XL, Sroka R, Razansky D. Volumetric optoacoustic temperature mapping in photothermal therapy. Sci Rep 2017;7(1):9695.
35. Landa FJO, Deán-Ben XL, Montero de Espinosa F, Razansky D. Noncontact monitoring of incision depth in laser surgery with air-coupled ultrasound transducers. Opt Lett 2016;41(12):2704-2707. https://doi.org/10.1364/ OL.41.002704. 06/15 2016.

36. Gaja H, Liou F. Defects monitoring of laser metal deposition using acoustic emission sensor. Int J Adv Manuf Technol 2017;90(1-4):561-574

37. Shevchik SA, Kenel C, Leinenbach C, Wasmer K. Acoustic emission for in situ quality monitoring in additive manufacturing using spectral convolutional neural networks. Addit Manuf 2018;21:598-604. https://doi.org/10.1016/j. addma.2017.11.012. 05/01/2018.

38. Gaja H, Liou F. Defect classification of laser metal deposition using logistic regression and artificial neural networks for pattern recognition. Int $\mathrm{J}$ Adv Manuf Technol 2018; 94(1-4):315-326.

39. Jaeger M, Schüpbach S, Gertsch A, Kitz M, Frenz M. Fourier reconstruction in optoacoustic imaging using truncated regularized inverse k-space interpolation. Inverse Problems 2007;23(6):S51-S63.

40. Mehta SS, Lingayat NS. Biomedical signal processing using SVM. In: 2007 IET-UK International Conference on Information and Communication Technology in Electrical Sciences (ICTES 2007). 2007. pp 527-532.

41. Dehmeshki J, Chen J, Casique MV, Karakoy M. Classification of lung data by sampling and support vector machine. In: The 26th Annual International Conference of the IEEE Engineering in Medicine and Biology Society. Vol 2. 2004. pp 3194-3197.

42. Memarian N, Alirezaie J, Babyn P. Computerized detection of lung nodules with an enhanced false positive reduction scheme. In: 2006 International Conference on Image Processing. 2006. pp 1921-1924. https://doi.org/10.1109/ICIP. 2006.313144

43. Guenther FH. Neural networks: Biological models and applications. In: Smelser NJ, Baltes PB, editors. International Encyclopedia of the Social \& Behavioral Sciences. Oxford: Pergamon; 2001. pp 10534-10537.

44. Goodfellow I, Bengio Y, Courville A. Deep Learning. London, England: MIT Press; 2016.

45. Andrearczyk V, Whelan PF. Chapter 4-Deep learning in texture analysis and its application to tissue image classification. In: Depeursinge A, Al-Kadi OS, Mitchell JR, editors. Biomedical Texture Analysis. London, England: Academic Press; 2017. pp 95-129.

46. Alperovich Z, Yamin G, Elul E, Bialolenker G, Ishaaya AA. In situ tissue classification during laser ablation using acoustic signals. J Biophotonics 2019;12:e201800405.

47. Brereton RG. The Mahalanobis distance and its relationship to principal component scores. J Chemom 2015;29(3): 143-145. https://doi.org/10.1002/cem.2692

48. Alvarez-Arenas TEG. Acoustic impedance matching of piezoelectric transducers to the air. IEEE Trans Ultrason Eng 2004;51(5):624-633. https://doi.org/10.1109/TUFFC. 2004.1320834

49. Álvarez-Arenas TG, Camacho J. Air-coupled and resonant pulse-echo ultrasonic technique. Sensors 2019;19(10):2221.

50. Lever J, Krzywinski M, Altman N. Principal component analysis. Nat Methods 2017;14:641-642. https://doi.org/10. 1038/nmeth.4346. 06/29/online

51. Bishop CM. Pattern Recognition and Machine Learning. London, England: Springer Science+ Business Media; 2006.

52. Lehman J, Risi S, Clune J. Creative generation of 3D objects with deep learning and innovation engines. In: Proceedings of the 7th International Conference on Computational Creativity. 2016.

53. Auria L, Moro RA. Support vector machines (SVM) as a technique for solvency analysis. 2008.

54. Dupont A, Caminat P, Bournot P, Gauchon J. Enhancement of material ablation using 248, 308, 532, $1064 \mathrm{~nm}$ laser pulse with a water film on the treated surface. J Appl Phys 1995;78(3):2022-2028.

55. Hyun Wook K, Ho L, Shaochen C, Welch AJ. Enhancement of bovine bone ablation assisted by a transparent liquid layer on a 
target surface. IEEE J Quantum Electron 2006;42(7):633-642. https://doi.org/10.1109/JQE.2006.875867

56. Manikanta E, Vinoth Kumar L, Venkateshwarlu P, Leela C, Kiran PP. Effect of pulse duration on the acoustic frequency emissions during the laser-induced breakdown of atmospheric air. Appl Opt 2016;55(3):548-555. https://doi.org/10. 1364/AO.55.000548. 01/20 2016

57. Yuldashev P, Karzova M, Khokhlova V, Ollivier S, BlancBenon P. Mach-Zehnder interferometry method for acoustic shock wave measurements in air and broadband calibration of microphones. J Acoust Soc Am 2015;137(6):3314-3324. https://doi.org/10.1121/1.4921549
58. Curzon M, Featherstone J. Chemical composition of enamel. In Lazzan EP, editor. Handbook of Experimental Aspects of Ora Biochemistry. Boca Raton, FL: CRC Press; 1983. pp 123-135.

59. Apel C, Meister J, Ioana R, Franzen R, Hering P, Gutknecht N The ablation threshold of Er:YAG and Er:YSGG laser radiation in dental enamel. Lasers Med Sci 2002;17(4):246-252.

60. Le T, Nguyen V, Nguyen TD, Phung D. Nonparametric budgeted stochastic gradient descent. In: Artificial Intelligence and Statistics. 2016. pp 654-572.

61. Jolliffe IT, Cadima J. Principal component analysis: A review and recent developments. Philos Trans R Soc A 2016; 374(2065):20150202. 